\title{
Whole-Body Biodistribution and Radiation Dosimetry of the New Cardiac Tracer 99mTc-N-DBODC
}

\author{
Corrado Cittanti ${ }^{1}$, Licia Uccelli ${ }^{1}$, Micol Pasquali ${ }^{1}$, Alessandra Boschi ${ }^{1}$, Claudia Flammia ${ }^{1}$, Elisa Bagatin ${ }^{1}$, \\ Massimiliano Casali ${ }^{1}$, Michael G. Stabin ${ }^{2}$, Luciano Feggi ${ }^{3}$, Melchiore Giganti ${ }^{1}$, and Adriano Duatti ${ }^{1}$ \\ ${ }^{1}$ Laboratory of Nuclear Medicine and Nuclear Medicine Section, Department of Radiological Sciences, University of Ferrara, Ferrara, \\ Italy; ${ }^{2}$ Department of Radiology and Radiological Sciences, Vanderbilt University, Nashville, Tennessee; and ${ }^{3}$ Nuclear Medicine Unit, \\ Department of Diagnostic Imaging and Laboratory Medicine, S. Anna University Hospital, Ferrara, Italy
}

Our purpose was to evaluate the safety profile and biodistribution behavior in healthy human volunteers of the new myocardial perfusion tracer bis[(dimethoxypropylphosphanyl)ethyl]ethoxyethylamine $N, N^{\prime}$-bis(ethoxyethyl)dithiocarbamato nitrido technetium (V) ( $\left.{ }^{99 m} \mathrm{Tc}-\mathrm{N}-\mathrm{DBODC}\right)$. Methods: Ten healthy male volunteers were injected with ${ }^{99 m} \mathrm{Tc}-\mathrm{N}-\mathrm{DBODC}$ under both stress and rest conditions. Anterior and posterior planar $\gamma$-camera images were collected at 5, 30, 60, 240, and 1,440 min after injection, with organ uptake quantified by region-of-interest analysis. Tracer kinetics in body fluids were determined by collecting blood and urine samples at different time points. Results: After injection, ${ }^{99 m T c-}$ $\mathrm{N}$-DBODC showed significant accumulation in the myocardium and prolonged retention. Under rest conditions, uptake in the heart, lungs, and liver at 5 min after injection was $1.67 \% \pm$ $0.13 \%, 1.16 \% \pm 0.07 \%$, and $10.85 \% \pm 1.72 \%$, respectively, of administered activity. Under stress conditions, heart uptake was significantly higher $(2.07 \% \pm 0.22 \%)$. Radioactivity in the liver decreased to $3.64 \% \pm 0.98 \%$ and $2.37 \% \pm 0.48 \%$ at 60 and $240 \mathrm{~min}$, respectively, after injection. This rapid liver clearance led to favorable heart-to-liver ratios, reaching values of $0.74 \pm 0.13$ at rest and $1.26 \pm 0.28$ during exercise $60 \mathrm{~min}$ after tracer administration. Radiation dose estimates were comparable to those obtained with other myocardial perfusion cationic compounds. Conclusion: The high uptake in the myocardium and the fast liver washout of 99mTc-N-DBODC will allow SPECT images of the left ventricle to be acquired early and with excellent quality.

Key Words: cardiac perfusion imaging; monocationic complexes; nitrido complexes; ${ }^{99 m T c}$

J Nucl Med 2008; 49:1299-1304

DOI: 10.2967/jnumed.108.053132

$\mathbf{P}$ reviously, we described a new class of asymmetric, monocationic nitrido ${ }^{99 \mathrm{~m}} \mathrm{Tc}$ complexes $(1,2)$. The biodistribution behavior of the derivative bis[(dimethoxypropylphosphanyl)-

Received Apr. 4, 2008; revision accepted May 8, 2008.

For correspondence or reprints contact: Adriano Duatti, Laboratory of Nuclear Medicine, Department of Radiological Sciences, University of Ferrara, Via L. Borsari, 46, 44100 Ferrara, Italy.

E-mail: dta@unife.it

COPYRIGHT @ 2008 by the Society of Nuclear Medicine, Inc. ethyl] ethoxyethylamine $N, N^{\prime}$-bis(ethoxyethyl)dithiocarbamato nitrido technetium(V) $\left({ }^{99} \mathrm{~m}\right.$ Tc-N-DBODC; Fig. 1) was studied extensively in rats $(3,4)$ and dogs $(5)$ and compared with that of the commercial radiopharmaceuticals ${ }^{99 \mathrm{~m}} \mathrm{Tc}$-sestamibi (Cardiolite; Bristol-Myers Squibb Medical Imaging, Inc.) and ${ }^{99 \mathrm{~m} T c-}$ tetrofosmin (Myoview; GE Healthcare). These results in animals showed that heart uptake of ${ }^{99 \mathrm{~m} T c-N-D B O D C}$ and washout from blood and lungs, as well as kinetic behavior as determined in dogs, are similar to those of the other 2 cardiac agents (5). However, a dramatic difference was observed in liver washout, which was remarkably rapid and quantitative. In particular, at 60 min after injection in rats, the heart-to-liver ratio of ${ }^{99 \mathrm{~m}} \mathrm{Tc}-\mathrm{N}-$ DBODC was approximately 10 times higher than that of ${ }^{99 \mathrm{~m} T c-}$ sestamibi and ${ }^{99 m}$ Tc-tetrofosmin $(2-4)$. On the basis of these promising results, this study was designed to evaluate the safety profile and the biodistribution of ${ }^{99 \mathrm{~m}} \mathrm{Tc}-\mathrm{N}-\mathrm{DBODC}$ in healthy human volunteers under both stress and rest conditions.

\section{MATERIALS AND METHODS}

\section{Study Population}

The study group included 10 healthy male volunteers (mean age $\pm \mathrm{SD}, 40.6 \pm 10.2 \mathrm{y}$; range, 29-54 y), with no risk factors or symptoms of cardiovascular disease and with normal findings on a resting 12-lead electrocardiogram and standard biologic tests.

The local Ethics Committee approved this study. All subjects read the experimental protocol and gave written informed consent.

\section{Preparation of ${ }^{99 m} \mathrm{Tc}-\mathrm{N}-\mathrm{DBODC}$}

The ligand bis[(dimethoxypropylphosphanyl)ethyl]ethoxyethylamine (PNP5) was donated by Nihon Medi-Physics. The ligand $N, N^{\prime}$-bis(ethoxyethyl)dithiocarbamato sodium salt (DBODC) was purchased from Alchemy. Stannous chloride dihydrate, succinic dihydrazide, $\gamma$-cyclodextrin, and ethylenediaminetetraacetic acid were obtained from Sigma-Aldrich. In all procedures, ethanol, water, and saline suitable for injection were used. Before use, SepPak cartridges (Waters) were washed with $5.0 \mathrm{~mL}$ of sterile water and then steam sterilized.

The compound ${ }^{99 \mathrm{~m}} \mathrm{Tc}-\mathrm{N}-\mathrm{DBODC}$ was prepared using a 2-vial (A and B) freeze-dried kit formulation. Vial A contained $5.0 \mathrm{mg}$ of succinic dihydrazide, $0.1 \mathrm{mg}$ of $\mathrm{SnCl}_{2} \cdot 2 \mathrm{H}_{2} \mathrm{O}, 5.0 \mathrm{mg}$ of ethylenediaminetetraacetic acid, and $1.0 \mathrm{~mL}$ of a phosphate buffer $(0.1$ 
FIGURE 1. Molecular structure of ${ }^{99 m}$ Tc-N-DBODC.

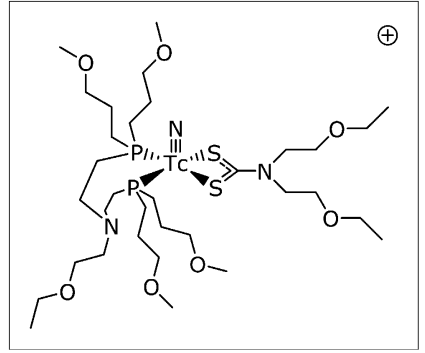

mol $\mathrm{dm}^{-3}$ ). Vial B contained $3.5 \mathrm{mg}$ of $\gamma$-cyclodextrin, $3.5 \mathrm{mg}$ of PNP5, and $3.5 \mathrm{mg}$ of DBODC.

Freshly eluted ${ }^{99 \mathrm{~m}} \mathrm{Tc}-\left[\mathrm{TcO}_{4}\right] \mathrm{Na}(0.37-11.1 \mathrm{GBq})$ was added to vial $\mathrm{A}$, and the solution was left to stand at room temperature for $30 \mathrm{~min}$. Then, $1.750 \mathrm{~mL}$ of saline were added to vial $\mathrm{B}$, and after complete dissolution of its content, $1.0 \mathrm{~mL}$ of the resulting solution was withdrawn with a syringe and added to vial A. Finally, this vial was placed in a heating block for $15 \mathrm{~min}$.

Quality control was performed as described elsewhere (2-4). The yield was $96 \% \pm 2.0 \%$. Radiochemical purity after purification was $99 \% \pm 0.8 \%$.

The final compound was purified of excess reagents using the following procedure. All operations were performed in a class A isolator equipped with a class B inlet/outlet prefiltering chamber. A reversed-phase $\mathrm{C} 18$ cartridge (Sep-Pak) was activated with $5.0 \mathrm{~mL}$ of absolute ethanol followed by $5.0 \mathrm{~mL}$ of sterile deionized water. The compound ${ }^{99 \mathrm{~m}} \mathrm{Tc}-\mathrm{N}-\mathrm{DBODC}$ was withdrawn from the reaction vial with a $10-\mathrm{mL}$ syringe, passed through the activated cartridge, and successively washed with $10.0 \mathrm{~mL}$ of sterile deionized water and $3.0 \mathrm{~mL}$ of a mixture of ethanol and water $(80: 20, \mathrm{v} / \mathrm{v})$. Activity was recovered from the cartridge with $1.0 \mathrm{~mL}$ of ethanol and saline $(90: 10, \mathrm{v} / \mathrm{v})$. A cation-exchange cartridge (Sep-Pak) was activated by $5.0 \mathrm{~mL}$ of sterile deionized water. The ${ }^{99 \mathrm{~m} T c-N-D B O D C}$ solution collected from the first purification step was withdrawn with a

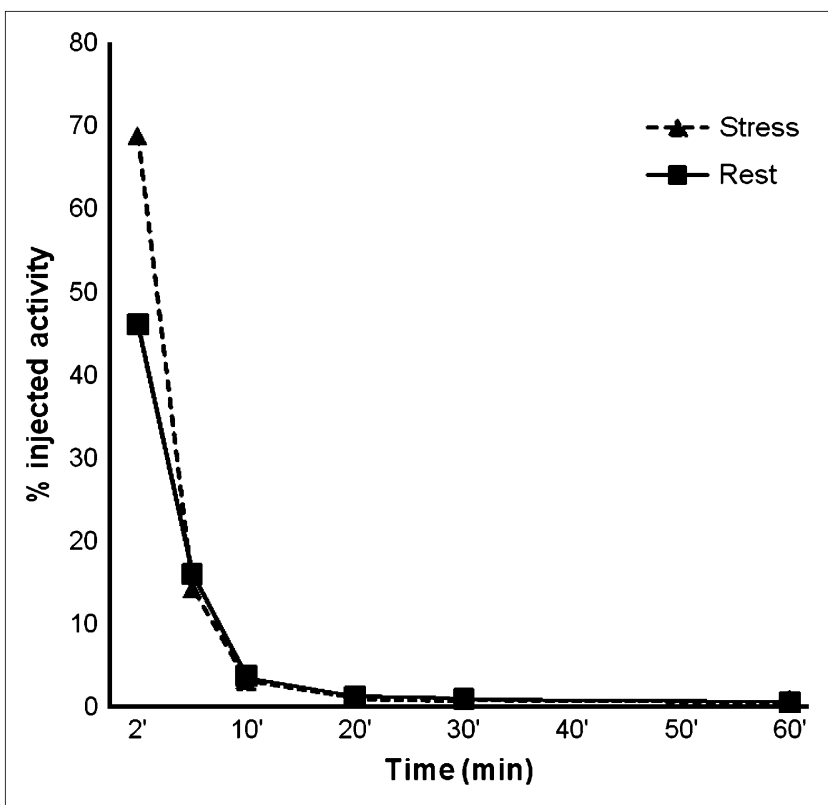

FIGURE 2. Blood clearance of ${ }^{99 \mathrm{~m} T c-N-D B O D C}$ under rest and stress conditions. Data (mean $\pm \mathrm{SD}$ ) are expressed as percentage injected activity in estimated total blood volume.
10.0-mL syringe and passed through the cartridge and then was successively washed with $2.5 \mathrm{~mL}$ of absolute ethanol and $1.0 \mathrm{~mL}$ of sterile deionized water. The activity was collected from the cartridge with $1.0 \mathrm{~mL}$ of ethanol and saline $(10: 90, \mathrm{v} / \mathrm{v})$. Sterile physiologic solution $(1.0 \mathrm{~mL})$ was added to the purified ${ }^{99 \mathrm{~m}} \mathrm{Tc}-\mathrm{N}-\mathrm{DBODC}$ solution and passed through a $0.22-\mu \mathrm{m}$ sterile filter (Millipore). Additional saline was further added to obtain a final volume of $3.0 \mathrm{~mL}$.

\section{Administration Protocol}

Five subjects were randomized to a rest group (A) and 5 to a peak stress group (B). All volunteers fasted before receiving an injection of ${ }^{99 \mathrm{~m}} \mathrm{Tc}-\mathrm{N}-\mathrm{DBODC}$ (mean injected activity, $377.3 \pm 50.7 \mathrm{MBq}$ ). The 2 groups did not significantly differ in age or whole-body characteristics (e.g., height, weight, and body surface area).

For patients undergoing exercise testing, a cycloergometric standardized protocol $(25 \mathrm{~W} \times 2 \mathrm{~min})$ was performed with monitoring of heart rate and rhythm, blood pressure, and electro-

\section{A}

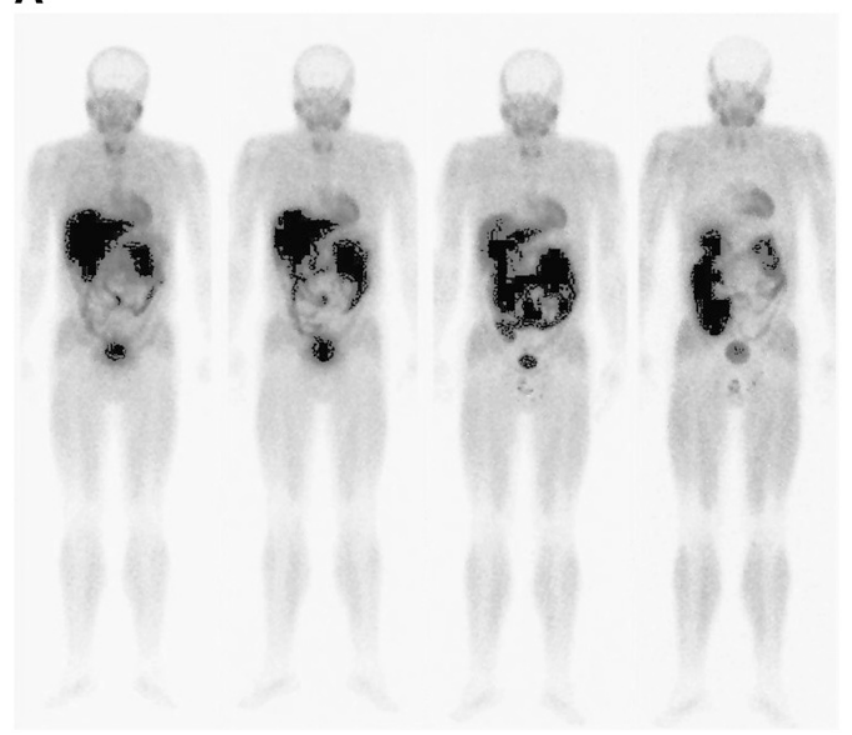

B
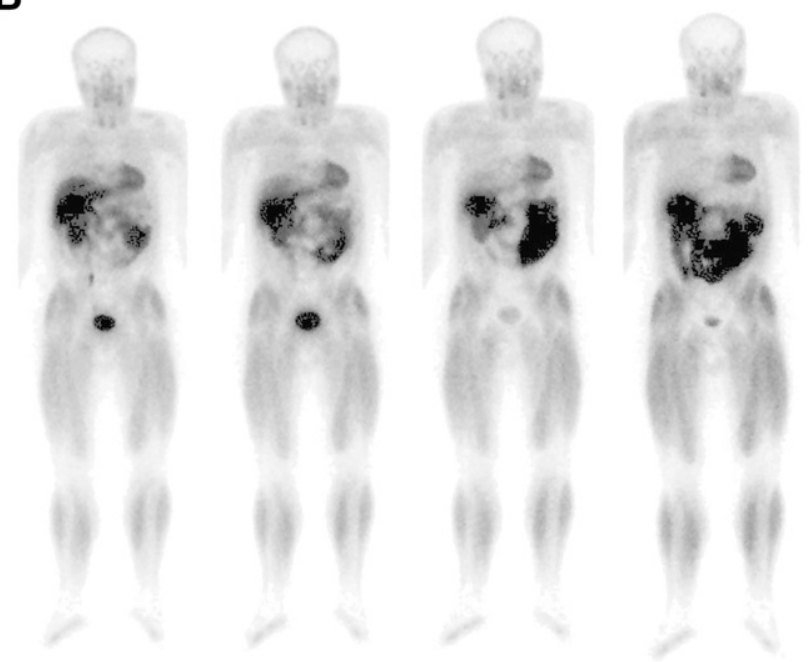

FIGURE 3. Planar whole-body images at different times (from left to right, $5,30,60$, and $240 \mathrm{~min}$ ) of biodistribution of ${ }^{99 \mathrm{mTC}} \mathrm{T}$ $\mathrm{N}$-DBODC in healthy volunteers under rest $(\mathrm{A})$ and stress $(\mathrm{B})$ conditions. 
cardiography. The test endpoint was established to be when $85 \%$ of the maximal predicted heart rate was achieved. The subjects were encouraged to continue exercising for a further minute after reaching the endpoint. All volunteers were allowed a light meal approximately $4 \mathrm{~h}$ after administration of the tracer.

\section{Safety Parameters}

Clinical examinations were performed $1 \mathrm{~h}$ before administration of ${ }^{99 \mathrm{~m}} \mathrm{Tc}-\mathrm{N}-\mathrm{DBODC}$ and $24 \mathrm{~h}$ after injection. Safety parameters such as vital signs (pulse, temperature, and blood pressure) were recorded $30 \mathrm{~min}$ before administration of the tracer and then at 5, 10, 15, 30, 60, 120, 240, 360, and 1,440 min after injection. A 12-lead electrocardiogram was recorded $30 \mathrm{~min}$ before injection and 360 and 1,440 min after injection.

Each volunteer was also followed up biologically. Blood samples were collected 5 min before injection and 30, 60, and 1,440 min after injection for determining standard parameters such as complete blood count, electrolytes, creatinine, urea, cholesterol, triglycerides, albumin, total serum protein, aspartate transaminase, alanine transaminase, $\gamma$-glutamyl-transferase, creatine phosphokinase, and alkaline phosphatase. Similarly, urine samples were recovered and analyzed to test for any sign of nephrotoxicity.

\section{Pharmacokinetic Studies}

In order to assess blood clearance of ${ }^{99 \mathrm{~m} T c-N-D B O D C}$ and its radioactive metabolites, serial venous sampling was performed through a cannula. Blood samples were taken 5 min before injection (baseline); 2, 10, 20, and 30 min after injection; and 60, 120, 240, 360, and 1,440 min after injection. Duplicate whole-blood and plasma samples were processed for counting. The results were expressed as percentage injected activity after correction for background and radionuclide decay.

Urine samples were collected during the $24 \mathrm{~h}$ after injection using disposable plastic containers. Urine collection and radioactivity measurements were grouped by interval after injection (0-2, $2-4,4-8,8-12$, and 12-24 h), and the results were expressed as percentage injected activity after correction for radionuclide decay.

\section{Biodistribution Studies}

Biodistribution was studied by acquiring anterior and posterior whole-body images 5, 30, 60, 240 and 1,440 min after injection using a dual-head rotating $\gamma$-camera (Vertex; Philips). The photopeak was centered at $140 \mathrm{keV}$ in a $20 \%$-width energy window using low-energy, high-resolution collimators.

Regions of interest were drawn manually over the images to identify the heart, lungs, liver, gallbladder, kidneys, salivary glands, thyroid, and spleen. Background regions of interest were drawn at a suitable area near the region of interest and subtracted to correct the conjugate-view counting rates for the contribution of the surrounding activity. Specifically, heart-to-liver ratios were calculated by selecting regions of interest on the whole heart and on the whole upper left lobe of the liver. All results were expressed as percentage injected activity after correction for ${ }^{99 \mathrm{~m}} \mathrm{Tc}$ decay.

\section{Dosimetry}

The effective attenuation coefficient, $\mu$, was determined for the radionuclide emissions in a scattering medium. The system calibration factor, $\mathrm{C}$, expressed as (counts $\times$ imaging time)/activity, was

TABLE 1

Biodistribution Results in Healthy Human Volunteers*

\begin{tabular}{|c|c|c|c|c|c|}
\hline \multirow[b]{2}{*}{ Organ } & \multicolumn{5}{|c|}{ Time after injection (min) } \\
\hline & 5 & 30 & 60 & 240 & 1,440 \\
\hline \multicolumn{6}{|l|}{ Heart } \\
\hline Rest & $1.67 \pm 0.13$ & $1.55 \pm 0.09$ & $1.52 \pm 0.06$ & $1.34 \pm 0.09$ & $0.50 \pm 0.02$ \\
\hline Exercise & $2.07 \pm 0.22$ & $2.01 \pm 0.01$ & $1.89 \pm 0.04$ & $1.54 \pm 0.09$ & $0.68 \pm 0.05$ \\
\hline \multicolumn{6}{|l|}{ Lung } \\
\hline Rest & $1.16 \pm 0.07$ & $1.11 \pm 0.08$ & $1.04 \pm 0.09$ & $0.49 \pm 0.12$ & $0.26 \pm 0.09$ \\
\hline Exercise & $1.12 \pm 0.21$ & $1.06 \pm 0.28$ & $0.91 \pm 0.40$ & $0.39 \pm 0.07$ & $0.15 \pm 0.05$ \\
\hline \multicolumn{6}{|l|}{ Liver } \\
\hline Rest & $10.85 \pm 1.72$ & $6.52 \pm 1.41$ & $4.31 \pm 1.19$ & $2.85 \pm 0.62$ & $0.36 \pm 0.16$ \\
\hline Exercise & $6.35 \pm 1.27$ & $3.93 \pm 0.98$ & $2.96 \pm 0.76$ & $1.89 \pm 0.33$ & $0.29 \pm 0.08$ \\
\hline \multicolumn{6}{|l|}{ Gallbladder } \\
\hline Rest & $3.93 \pm 1.36$ & $4.15 \pm 1.68$ & $5.09 \pm 2.37$ & $4.98 \pm 3.06$ & $1.61 \pm 1.12$ \\
\hline Exercise & $3.38 \pm 0.81$ & $4.05 \pm 1.13$ & $4.27 \pm 1.70$ & $4.16 \pm 1.00$ & $1.47 \pm 0.82$ \\
\hline \multicolumn{6}{|l|}{ Kidney } \\
\hline Rest & $5.06 \pm 1.66$ & $4.25 \pm 1.19$ & $4.92 \pm 1.87$ & $3.84 \pm 0.78$ & $0.99 \pm 0.20$ \\
\hline Exercise & $5.76 \pm 0.80$ & $4.91 \pm 0.50$ & $4.80 \pm 0.81$ & $4.16 \pm 0.41$ & $1.15 \pm 0.14$ \\
\hline \multicolumn{6}{|c|}{ Salivary glands } \\
\hline Rest & $0.22 \pm 0.02$ & $0.20 \pm 0.03$ & $0.20 \pm 0.03$ & $0.17 \pm 0.04$ & $0.10 \pm 0.03$ \\
\hline Exercise & $0.20 \pm 0.05$ & $0.18 \pm 0.04$ & $0.17 \pm 0.03$ & $0.13 \pm 0.02$ & $0.10 \pm 0.03$ \\
\hline \multicolumn{6}{|l|}{ Thyroid } \\
\hline Rest & $0.64 \pm 0.12$ & $0.54 \pm 0.10$ & $0.53 \pm 0.12$ & $0.42 \pm 0.07$ & $0.17 \pm 0.04$ \\
\hline Exercise & $0.52 \pm 0.12$ & $0.43 \pm 0.11$ & $0.44 \pm 0.13$ & $0.35 \pm 0.09$ & $0.20 \pm 0.06$ \\
\hline \multicolumn{6}{|l|}{ Spleen } \\
\hline Rest & $0.80 \pm 0.32$ & $0.60 \pm 0.22$ & $0.48 \pm 0.11$ & $0.32 \pm 0.06$ & $0.17 \pm 0.04$ \\
\hline Exercise & $0.76 \pm 0.23$ & $0.61 \pm 0.16$ & $0.54 \pm 0.18$ & $0.42 \pm 0.13$ & $0.19 \pm 0.10$ \\
\hline
\end{tabular}

*Data are percentage injected activity per organ. 
determined and used to convert counts in activity. These values were subsequently used with planar imaging data to calculate internal dose estimates for each healthy volunteer. The OLINDA/ EXM program (6) was applied for radiation dosimetry calculations using the ICRP 30 gastrointestinal model for representing the fraction of activity entering the small intestine and the voidingbladder model (7) for determining the urinary excretion counts.

\section{Statistical Analysis}

All results are expressed as mean \pm SD. The statistical significance of differences was assessed using the Student $t$ test or the paired $t$ test where indicated, with a $P$ value of less than 0.05 being considered significant. Individual safety data for each healthy volunteer were also analyzed to determine whether clinically significant drug-related trends could be detected by comparing pre- and postinjection values for each monitored parameter and recording any shift from normal to abnormal ranges.

\section{RESULTS}

\section{Safety Data}

Safety parameters measured up to $24 \mathrm{~h}$ after injection of ${ }^{99 m}$ Tc-N-DBODC revealed no clinically significant longterm drug-related changes. Moreover, no significant adverse reactions were reported, and no modification of vital signs or of other biologic parameters was detected.

\section{Pharmacokinetic Data}

Blood clearance of ${ }^{99} \mathrm{~m}$ Tc-N-DBODC was fast; 10 min after injection, only $3.51 \% \pm 0.85 \%$ of the injected activity remained in the whole-blood volume (Fig. 2). After exercise, activity in the blood was significantly higher than at rest only at $2 \mathrm{~min}$ after injection $(68.81 \% \pm 9.48 \%$ vs. $46.06 \% \pm 11.28 \%, P<0.01$ ) (Fig. 2). In the interval from 5 min to 1,440 min, no significant differences were detected in blood clearance between groups $\mathrm{A}$ and $\mathrm{B}(P=$ not statistically significant). Plasmatic clearance of the compound followed blood clearance kinetics closely.

Cumulative urine radioactivity, collected over $24 \mathrm{~h}$, was $21.42 \% \pm 6.48 \%$ of the injected activity. The rate of urinary clearance during the first $2 \mathrm{~h}$ was about $50 \%$ higher in group A than in group B $(14.30 \% \pm 6.76 \%$ vs. $9.76 \% \pm 1.72 \%, P<$ 0.05). However, after $24 \mathrm{~h}$ after injection, an equivalent amount of the injected activity of ${ }^{99} \mathrm{~m}$ Tc-N-DBODC and of its radioactive metabolites was excreted into the urine under both conditions $(22.18 \% \pm 8.49 \%$ at rest vs. $20.47 \% \pm$ $3.69 \%$ after exercise, $P=$ not statistically significant).

\section{Biodistribution}

Good-quality images of the heart were obtained in all healthy volunteers between $5 \mathrm{~min}$ and several hours after tracer administration. In early images acquired both at rest and during stress, qualitative analyses revealed no significant overlap between tracer accumulation in the inferior wall of the left ventricle and in the subdiaphragmatic region.

A quantitative analysis of serial whole-body images showed that uptake in the heart, lungs, and liver at $5 \mathrm{~min}$ after injection was $1.87 \% \pm 0.18 \%, 1.14 \% \pm 0.14 \%$, and $8.60 \% \pm 1.50 \%$, respectively, of administered activity.
Radioactivity in the liver decreased rapidly to $3.64 \% \pm$ $0.98 \%$ and $2.37 \% \pm 0.48 \%$ at 60 and $240 \mathrm{~min}$, respectively, after injection. In contrast, a slow washout of myocardial activity was observed $(1.44 \% \pm 0.09 \%$ at $240 \mathrm{~min}$ after injection). Moreover, after an ergometric stress test, heart uptake was significantly higher than that observed under rest conditions $(2.07 \% \pm 0.22 \%$ vs. $1.67 \% \pm 0.13 \%, P<$ 0.01 ), and as expected, accumulation of activity by skeletal muscles was sharply enhanced during exercise.

Uptake by the salivary glands and thyroid was low both at rest and during stress, and only a slow washout was observed during the first $240 \mathrm{~min}$. Uptake by the kidneys averaged 4\%$6 \%$ of the injected activity at all time points within the first $240 \mathrm{~min}$, and rest and stress data did not significantly differ.

Representative planar whole-body images of the biodistribution of ${ }^{99 \mathrm{~m}} \mathrm{Tc}-\mathrm{N}-\mathrm{DBODC}$ in 2 healthy volunteers are shown in Figure 3, and detailed biodistribution results are reported in Table 1. Heart-to-lung ratios at $5 \mathrm{~min}$ after injection were high $(2.17 \pm 0.20$ at rest; $2.55 \pm 0.29$ after exercise) and improved only slightly over time (Fig. 4A). No statistically significant differences were noticed between ratios at rest and during exercise during the first 240 min. Heart-to-liver ratios (Fig. 4B) were initially moderate $(0.33 \pm 0.07$ at rest; $0.66 \pm 0.19$ after exercise $)$ but rapidly increased during the first hour $(0.74 \pm 0.13$ at rest; $1.26 \pm$ 0.28 after exercise). Ratios from exercise studies were
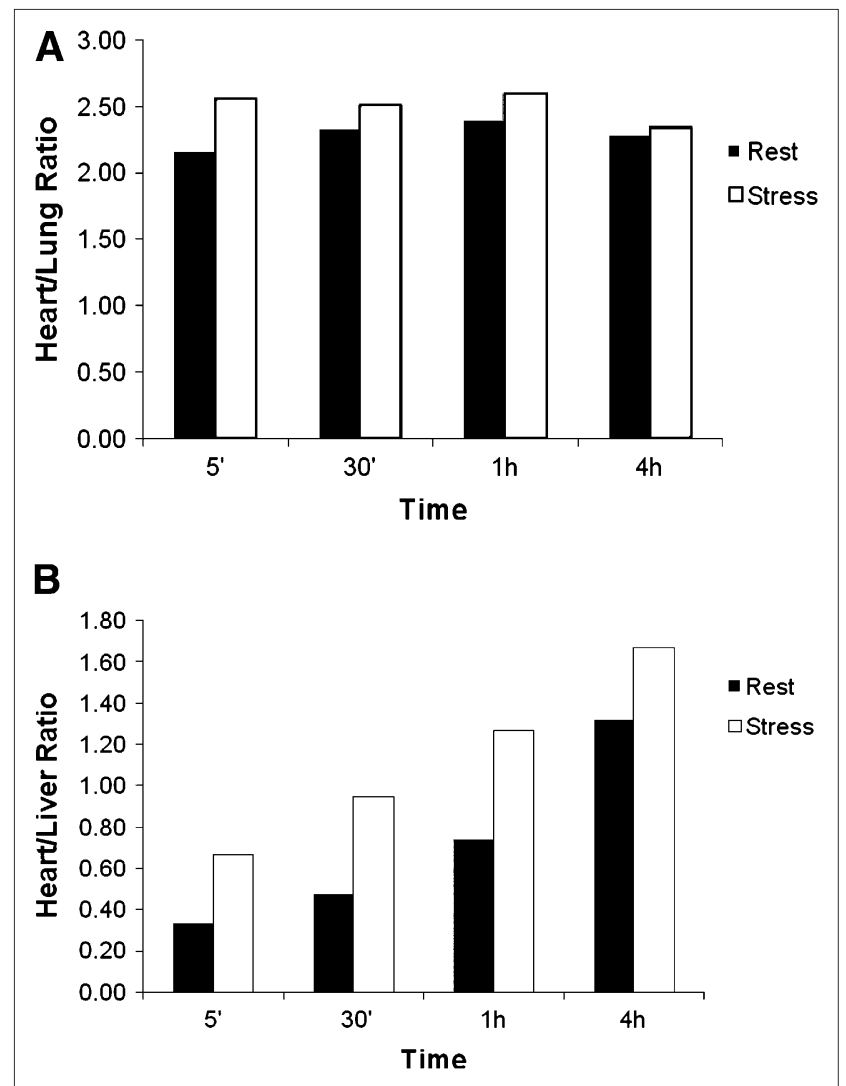

FIGURE 4. Heart-to-lung (A) and heart-to-liver (B) ratios measured at different times for $99 \mathrm{mTc}-\mathrm{N}-\mathrm{DBODC}$ under both stress and rest conditions. 
significantly higher $(P<0.01)$ than those from resting studies at all times up to $60 \mathrm{~min}$ after injection (Fig. 4).

\section{Dosimetry}

The results of dosimetry calculations are presented in Table 2. Radiation dose estimates were in the same range as those reported for other ${ }^{99 \mathrm{~m}} \mathrm{Tc}$ myocardial perfusion agents (8-11) and, therefore, lower than for ${ }^{201} \mathrm{Tl}$ (12). Gallbladder, intestines, kidneys, and bladder received the highest dose, as these organs are involved in the primary excretory pathway of ${ }^{99 \mathrm{~m}}$ Tc-N-DBODC. Among them, the gallbladder wall and kidneys absorbed the highest activity. There were no relevant differences between rest and stress conditions.

\section{DISCUSSION}

An ideal cardiac perfusion imaging agent has long been sought in nuclear cardiology. The commercial agents ${ }^{99 \mathrm{~m}} \mathrm{Tc}-$ sestamibi and ${ }^{99 \mathrm{~m}}$ Tc-tetrofosmin do not have all the traits commonly considered to be characteristic of an ideal tracer. In particular, a high first-pass extraction, fast liver washout, and linear relationship between flow and tracer uptake are key indicators of ideal behavior. As previously reported $(13,14),{ }^{99 m}$ Tc-sestamibi and ${ }^{99 m}$ Tc-tetrofosmin do not ex- hibit optimal values for these parameters. Experience in this field has shown that it is not always possible to accommodate all these features in the same ${ }^{99 \mathrm{~m}} \mathrm{Tc}$-compound. Sometimes, certain properties of a given compound that prove beneficial in one aspect of quantitative imaging may be detrimental in others. For instance, it seems quite difficult to combine in the same ${ }^{99 m}$ Tc-complex a high first-pass extraction and fast liver washout. Such conflict was noted with the tracer ${ }^{99 \mathrm{~m}} \mathrm{Tc}$ N-NOET. The biologic behavior of this neutral compound showed the closest similarity to that of the cationic heart imaging agent ${ }^{201} \mathrm{Tl}(15-17)$ in high first-pass extraction and redistribution, but a high and persistent liver uptake was an important drawback of this tracer. Cationic compounds usually exhibit a moderate first-pass extraction and negligible redistribution, but liver uptake could be efficiently lowered through a convenient selection of ancillary chemical groups appended to the complex.

Among available commercial agents, ${ }^{99} \mathrm{~m}$ Tc-tetrofosmin exhibits the fastest liver washout and best approaches the ideal. To our knowledge, our study is the first reported evaluation of the human biodistribution properties of the monocationic cardiac perfusion imaging agent $99 \mathrm{~m} \mathrm{Tc}-\mathrm{N}-$ DBODC, which appears to represent a step further in the

TABLE 2

Radiation Dosimetry Estimates for ${ }^{99 m T c-N-D B O D C}$ in 10 Healthy Subjects

\begin{tabular}{|c|c|c|c|c|}
\hline \multirow[b]{2}{*}{ Organ } & \multicolumn{2}{|c|}{ Rest } & \multicolumn{2}{|c|}{ Stress } \\
\hline & $(\mathrm{mGy} / \mathrm{MBq}) \times 10^{2}$ & mGy/925 MBq & $(\mathrm{mGy} / \mathrm{MBq}) \times 10^{2}$ & mGy/925 MBq \\
\hline Adrenals & $0.55 \pm 0.10$ & $5.04 \pm 0.95$ & $0.55 \pm 0.01$ & $5.10 \pm 0.12$ \\
\hline Brain & $0.21 \pm 0.04$ & $1.98 \pm 0.39$ & $0.19 \pm 0.03$ & $1.74 \pm 0.31$ \\
\hline Breasts & $0.19 \pm 0.02$ & $1.74 \pm 0.21$ & $0.18 \pm 0.03$ & $1.65 \pm 0.26$ \\
\hline Gallbladder wall & $6.17 \pm 3.16$ & $57.07 \pm 29.24$ & $6.76 \pm 1.30$ & $62.49 \pm 12.07$ \\
\hline Lower large intestine wall & $1.11 \pm 0.23$ & $10.27 \pm 2.11$ & $1.31 \pm 0.37$ & $12.11 \pm 3.42$ \\
\hline Small intestine & $0.99 \pm 0.17$ & $9.12 \pm 1.55$ & $1.13 \pm 0.25$ & $10.45 \pm 2.34$ \\
\hline Stomach wall & $0.44 \pm 0.06$ & $4.08 \pm 0.52$ & $0.45 \pm 0.02$ & $4.16 \pm 0.23$ \\
\hline Upper large intestine wall & $1.56 \pm 0.32$ & $14.45 \pm 2.96$ & $1.86 \pm 0.53$ & $17.22 \pm 4.87$ \\
\hline Heart wall & $0.54 \pm 0.12$ & $4.96 \pm 1.07$ & $0.62 \pm 0.14$ & $5.74 \pm 1.31$ \\
\hline Kidneys & $3.31 \pm 1.54$ & $30.60 \pm 14.24$ & $3.66 \pm 0.75$ & $33.87 \pm 6.90$ \\
\hline Liver & $0.73 \pm 0.17$ & $6.75 \pm 1.56$ & $0.69 \pm 0.06$ & $6.37 \pm 0.52$ \\
\hline Lungs & $0.26 \pm 0.04$ & $2.43 \pm 0.38$ & $0.28 \pm 0.05$ & $2.55 \pm 0.47$ \\
\hline Muscle & $0.29 \pm 0.02$ & $2.67 \pm 0.20$ & $0.30 \pm 0.02$ & $2.79 \pm 0.22$ \\
\hline Ovaries & $0.61 \pm 0.07$ & $5.63 \pm 0.64$ & $0.66 \pm 0.10$ & $6.13 \pm 0.90$ \\
\hline Pancreas & $0.59 \pm 0.10$ & $5.45 \pm 0.92$ & $0.60 \pm 0.04$ & $5.53 \pm 0.34$ \\
\hline Red marrow & $0.33 \pm 0.04$ & $3.10 \pm 0.37$ & $0.34 \pm 0.02$ & $3.12 \pm 0.17$ \\
\hline Osteogenic cells & $0.75 \pm 0.07$ & $6.94 \pm 0.63$ & $0.72 \pm 0.08$ & $6.68 \pm 0.78$ \\
\hline Skin & $0.18 \pm 0.01$ & $1.64 \pm 0.13$ & $0.17 \pm 0.02$ & $1.59 \pm 0.19$ \\
\hline Spleen & $0.54 \pm 0.21$ & $5.02 \pm 1.90$ & $0.49 \pm 0.09$ & $4.53 \pm 0.79$ \\
\hline Testes & $0.26 \pm 0.02$ & $2.39 \pm 0.14$ & $0.25 \pm 0.03$ & $2.31 \pm 0.27$ \\
\hline Thymus & $0.26 \pm 0.02$ & $2.39 \pm 0.18$ & $0.25 \pm 0.04$ & $2.35 \pm 0.37$ \\
\hline Thyroid & $0.24 \pm 0.01$ & $2.23 \pm 0.13$ & $0.23 \pm 0.04$ & $2.11 \pm 0.37$ \\
\hline Urinary bladder wall & $1.36 \pm 0.21$ & $12.62 \pm 1.95$ & $1.39 \pm 0.18$ & $12.86 \pm 1.65$ \\
\hline Uterus & $0.59 \pm 0.05$ & $5.42 \pm 0.47$ & $0.62 \pm 0.06$ & $5.69 \pm 0.56$ \\
\hline \multirow[t]{2}{*}{ Total body } & $0.35 \pm 0.04$ & $3.23 \pm 0.33$ & $0.35 \pm 0.02$ & $3.26 \pm 0.20$ \\
\hline & $(\mathrm{mSv} / \mathrm{MBq}) \times 10^{-2}$ & $\mathrm{mSv} / 925 \mathrm{MBq}$ & $(\mathrm{mSv} / \mathrm{MBq}) \times 10^{-2}$ & $\mathrm{mSv} / 925 \mathrm{MBq}$ \\
\hline Effective dose equivalent & $1.09 \pm 0.23$ & $10.12 \pm 2.17$ & $1.19 \pm 0.07$ & $11.04 \pm 0.66$ \\
\hline Effective dose & $0.61 \pm 0.09$ & $5.66 \pm 0.79$ & $0.66 \pm 0.07$ & $6.08 \pm 0.69$ \\
\hline
\end{tabular}

Data are expressed as mean \pm SD. 
quest for an ideal tracer. Our results indicate that this tracer has the desirable characteristics of the highest heart uptake observed for a monocationic myocardial perfusion tracer and a fast and quantitative washout from nontarget organs surrounding the heart region. In particular, liver activity is almost completely eliminated into the intestine after $60 \mathrm{~min}$ after injection, with some residual activity remaining in the gallbladder. This behavior is significantly enhanced by exercise. Under these conditions, the heart region is quickly visualized at $5 \mathrm{~min}$ after injection, and liver activity appears to be significantly reduced also after $30 \mathrm{~min}$ after tracer administration. Concomitantly, another efficient elimination route is the kidneys. The absence of relevant background activity becomes particularly apparent after 240 min after injection (Fig. 3). Mean activity in the liver is rapidly exceeded by that in the myocardium, which thus becomes the most relevant localization in this area.

The radiation dosimetry calculations for ${ }^{99 \mathrm{~m}} \mathrm{Tc}-\mathrm{N}-\mathrm{DBODC}$ gave results comparable to data reported for other ${ }^{99 \mathrm{~m}} \mathrm{Tc}$ myocardial perfusion agents (8-11) and very favorable with respect to ${ }^{201} \mathrm{Tl}$. Thus, possible diagnostic use of the new cardiac agent would not increase the radiation risk for the patient.

The high myocardial uptake and quantitative liver washout result in favorable heart-to-liver ratios (Fig. 2). These ratios were calculated by applying a different procedure from that reported previously for other cardiac agents $(8,9,11)$, wherein only a tiny section of tissues surrounding the heart region was selected (8) or region-of-interest selection was not precisely defined (11). This procedure provided unequivocal results and is consistent with more accurate methods that have been described $(18,19)$.

\section{CONCLUSION}

The new monocationic tracer ${ }^{99 \mathrm{~m}} \mathrm{Tc}-\mathrm{N}-\mathrm{DBODC}$ is comparable to ${ }^{99 \mathrm{~m}} \mathrm{Tc}$-sestamibi (8) and ${ }^{99 \mathrm{~m}} \mathrm{Tc}$-tetrofosmin (9) in safety and radiation dosimetry. Although first-pass extraction and kinetic behavior of this agent appear to closely parallel those of the commercial radiopharmaceuticals (13), its high heart uptake and fast liver clearance allow early visualization of myocardial tissue, particularly under stress conditions. Therefore, ${ }^{99 \mathrm{~m}} \mathrm{Tc}-\mathrm{N}-\mathrm{DBODC}$ appears to be a promising myocardial perfusion tracer with improved imaging properties. For clinical applications, the rapid liver clearance may shorten the duration of imaging protocols and permit a more precise determination of defects in the inferoapical wall on myocardial images.

\section{ACKNOWLEDGMENTS}

We thank Dr. Napoleone Prandini, Dr. Massimo Roncali, Dr. Vincenzo De Biasi, and Laura Ferraguti for excellent technical assistance. This work was supported in part by a special research grant from the University of Ferrara.

\section{REFERENCES}

1. Bolzati C, Refosco F, Cagnolini A, et al. Synthesis, solution-state and solidstate structural characterization of monocationic nitrido heterocomplexes $[\mathrm{M}(\mathrm{N})(\mathrm{DTC})(\mathrm{PNP})]^{+}\left(\mathrm{M}={ }^{99} \mathrm{Tc}, \mathrm{Re} ; \mathrm{DTC}=\right.$ dithiocarbamate; PNP = heterodiphosphane). Eur J Inorg Chem. 2004;9:1902-1913.

2. Boschi A, Uccelli L, Bolzati C, et al. Synthesis and biological evaluation of monocationic asymmetric ${ }^{99 \mathrm{~m}} \mathrm{Tc}$-nitride heterocomplexes showing high heart uptake and improved imaging properties. J Nucl Med. 2003;44:806-814.

3. Boschi A, Bolzati C, Uccelli L, et al. A class of asymmetrical nitrido ${ }^{99 \mathrm{~m}} \mathrm{Tc}$ heterocomplexes as heart imaging agents with improved biological properties. Nucl Med Commun. 2002;23:689-693.

4. Hatada K, Riou LM, Ruiz M, et al. ${ }^{99 \mathrm{~m} T c-N-D B O D C 5}$, a new myocardial perfusion imaging agent with rapid liver clearance: comparison with ${ }^{99 \mathrm{~m}} \mathrm{Tc}-$ sestamibi and ${ }^{99 m}$ Tc-tetrofosmin in rats. J Nucl Med. 2004;45:2095-2101.

5. Hatada K, Ruiz M, Riou LM, et al. Biodistribution and myocardial uptake, washout, and redistribution kinetics of Tc-99m N-DBODC5 when injected during vasodilator stress in canine models of coronary stenoses. J Nucl Cardiol. 2006;13:779-790.

6. Stabin MG, Sparks RB, Crowe E. OLINDA/EXM: the second-generation personal computer software for internal dose assessment in nuclear medicine. J Nucl Med. 2005;46:1023-1027.

7. Foster DM. Developing and testing integrated multicompartment models to describe a single-input multiple-output study using the SAAM II software system. In: Clifford AJ, Müller H-G, eds. Mathematical Modeling in Experimental Nutrition. New York, NY: Plenum Press; 1998:59-78.

8. Wackers FJ, Berman DS, Maddahi J, et al. Technetium-99m hexakis 2-methoxyisobutyl isonitrile: human biodistribution, dosimetry, safety, and preliminary comparison to thallium-201 for myocardial perfusion imaging. J Nucl Med. 1989;30:301-311.

9. Higley B, Smith FW, Smith T, et al. Technetium-99m-1,2-bis[bis(2-ethoxyethyl)phosphino]ethane: human biodistribution, dosimetry and safety of a new myocardial perfusion imaging agent. J Nucl Med. 1993;34:30-38.

10. Rohe RC, Thomas SR, Stabin MG, et al. Biokinetics and dosimetry analysis in healthy volunteers for a two-injection (rest-stress) protocol of the myocardial perfusion imaging agent technetium 99m-labeled Q3. J Nucl Cardiol. 1995;2:395-404.

11. Vanzetto G, Fagret D, Pasqualini R, Mathieu JP, Chossat F, Machecourt J. Biodistribution, dosimetry, and safety of the myocardial perfusion agent ${ }^{99 m}$ TcNNOET in healthy volunteers. J Nucl Med. 2000;41:141-148.

12. ICRP. ICRP Publication 18: Radiation Dose to Patients from Radiopharmaceuticals. Oxford, U.K.: Pergamon Press; 1987:1-29.

13. Beller GA, Bergmann SR. Myocardial perfusion imaging agents: SPECT and PET. J Nucl Cardiol. 2004;11:71-86.

14. Jain D. Technetium-99m myocardial perfusion imaging agents. Semin Nucl Med. 1999;29:221-236.

15. Vanzetto G, Calnon DA, Ruiz M, et al. Myocardial uptake and redistribution of ${ }^{99 m}$ Tc-NNOET in dogs with either sustained coronary low flow or transient coronary occlusion: comparison with ${ }^{201} \mathrm{Tl}$ and myocardial blood flow. Circulation. 1997;96:2325-2331.

16. Fagret D, Marie PY, Brunotte F, et al. Myocardial perfusion imaging with technetium-99m-Tc-NOET: comparison with thallium-201 and coronary angiography. J Nucl Med. 1995;36:936-943.

17. Jeetley P, Sabharwal NK, Soman P, et al. Comparison between Tc-99m N-NOET and Tl-201 in the assessment of patients with known or suspected coronary artery disease. J Nucl Cardiol. 2004;11:664-672.

18. Stern S, Greenberg D, Corne R. Quantification of walking exercise required for improvement of dipyridamole thallium-201 image quality. J Nucl Med. 1992;33: 2061-2066.

19. Vitola JV, Brambatti JC, Caligaris F, et al. Exercise supplementation to dipyridamole prevents hypotension, improves electrocardiogram sensitivity, and increases heart-to-liver activity ratio on Tc-99m sestamibi imaging. $\mathrm{J} \mathrm{Nucl}$ Cardiol. 2001;8:652-659. 